\title{
Influencia de la parcialidad laboral en la viabilidad del sistema de pensiones español
}

\author{
Beatriz Rosado-Cebrián \\ Inmaculada Domínguez-Fabián
}

RESUMEN: El objetivo fundamental de este trabajo es el análisis de la viabilidad financiera del sistema contributivo de pensiones de jubilación español, tras las reformas adoptadas por la Ley 27/2011 y la Ley 23/2013, teniendo en cuenta el incremento del desempleo y de la parcialidad de los últimos años. La investigación se desarrolla a partir del estudio de trayectorias laborales "tipo", extraídas de las probabilidades de transición entre el empleo a tiempo completo, el empleo a tiempo parcial, el desempleo contributivo y la inactividad obtenidas a partir de la Encuesta de Condiciones de Vida (ECV2011-2014). El efecto que estas transiciones laborales tiene sobre la viabilidad financiera del sistema de pensiones público se mide a partir del Tanto Interno de Rendimiento (TIR).

PALABRAS CLAVE: Viabilidad, Parcialidad, Desempleo, Encuesta de Condiciones de Vida, Tanto Interno de Rendimiento, TIR.

CLAVES ECONLIT: G0, G2, H0.

Cómo citar este artículo / How to cite this article: ROSADO-CEBRIÁN, B. \& DOMÍNGUEZ-FABIÁN, I. (2017):

"Influencia de la parcialidad laboral en la viabilidad del sistema de pensiones español", CIRIEC-España, Revista de Economía Pública, Social y Cooperativa, 91, 235-266.

Correspondencia: Beatriz Rosado-Cebrián, Departamento de Economía Financiera y Contabilidad de la

Universidad de Extremadura, Centro Universitario de Plasencia, Avda. Virgen del Puerto, s/n. 10600, Plasencia (Cáceres), e-mail: brosadot@unex.es; Inmaculada Domínguez-Fabián, Departamento de Economía Financiera y Contabilidad de la Universidad de Extremadura, Facultad de Empresa, Finanzas y Turismo, Avda. de la Universidad s/n 10071, Cáceres, e-mail: idomingu@unex.es. 


\section{EXPANDED ABSTRACT}

\section{Influence of Part-Time Work on the Viability of the Spanish Pension System}

The sustainability problems faced by the Spanish pension system, which are caused by the increase in the number of pensioners without a generational replacement, causes a growing imbalance between the income obtained from those who contribute and the expenses of those who receive a pension. This fact has been aggravated by the economic crisis, mainly due to the fall in income from contributions and the increase in expenses due to contributory benefits.

In this context, the increase in the unemployment rate and longevity tends, on the one hand, to reduce the number of contributors and, on the other hand, to increase the number of pensions, putting the cash balance at risk, that is, the relationship between revenue and expenditure of the system. Therefore, the analysis of the relationship of the pension system and changes in the labour market becomes essential. The increase in unemployment and part-time work are elements that should be considered in the pension system analysis. This produces an interesting issue from the social and economic point of view, and it is necessary to cope with the changes that have taken place in the labour market and on retirement pensions, which are the most relevant in Spain, in terms of number and amount.

The main objective is the study of the effect that unemployment and part-time work have on the financial viability of the Spanish pension system. The analysis is carried out following the reforms introduced in 2011 and 2013, and as from Law 1/2014 of 28 February, for the protection of part-time workers and other urgent measures in the economic and social order. The research is based on the Living Conditions Survey (ECV, 2011-2014) of the INE (National Institute of Statistics), which provides the transition probabilities between full-time employment, part-time employment, contributory unemployment and inactivity, which are the different work "type" careers observed.

First, we estimated the transition probabilities between employment states provided by ECV: FullTime Employment (FT), Part-Time Employment (PT), Contributory Unemployment (U) and Inactivity (I), based on the stochastic model developed by Alonso and Rosado (2016).

Secondly, and in order to measure the sustainability of a pension system, the Internal Rate of Return (IRR) is used, as by following Devesa, Lejárraga and Vidal (2002), this method was chosen to analyse the financial equilibrium of the system through individuals "type" or representative contributors. The relationship between the IRR and the sustainability of a pay-as-you-go system derives from Samuelson's 
(1958) proposition, who claims that a pay-as-you-go pension system is financially sustainable in the long-term if its IRR does not exceed the real growth rate of the average wage plus the growth rate of the contributing population or, what is the same, does not exceed the growth of the fiscal base of the system which, in the long-term, is equal to the real GDP growth rate

Considering the results, it can be seen how a 25 year-old individual in 2016, that enters the labour market this year, and retires at the legal age, obtains a IRR of $2.62 \%$, in the case of maintaining Fulltime Employment throughout his working life. The same individual in the case of maintaining Part-time Employment throughout his working life obtains a probable IRR of $3.35 \%$, which is higher than the longterm Spanish economic growth projected by the European Commission (3\%).

In the analysis of work careers according to the percentage of episodes of Full-time Employment, Part-Time Employment, Contributory Unemployment and Inactivity throughout a working life, it is observed that as the episodes of Part-time work, of Contributory Unemployment and of Inactivity increase throughout the working life of the analysed individual, the profitability provided by the system increases above the solvency limit. In the case of changing to Unemployment, the probable IRR increases to $3.25 \%$ for Full-Time Employment and up to $3.62 \%$, in the case of changing to Part-time work. This shows the gap between the contributions made by social contributions throughout a working life and retirement pensions that will be received in the future.

In the case of work careers that start with Unemployment and change to Employment, the profitability provided by the system improves, while in the cases where there are Inactivity episodes throughout a working life, the probable IRR increases beyond the considered sustainability limit.

It should be mentioned that the results obtained for the probable IRR are valid for different "types" of possible work careers for a 25-year old individual in 2016, for whom the transition probabilities between employment states have been applied, which we assume will remain at these levels in the coming years.

In this sense, we are aware that this is a pessimistic view on the evolution of employment, since it is considered that the transition probabilities obtained during the economic crisis are maintained over time, so as the transition improves to Full-time Employment, IRR results and therefore, the viability of the system will improve, although predictably the desired financial balance will not be achieved. In spite of knowing this main research limitation, we understand that the model provides relevant results and conclusions in relation to the inclusion of part-time work and unemployment in the working lives of future pensioners.

From the results obtained, it can be concluded that as episodes of Part-Time Employment, Contributory Unemployment and Inactivity in the working lives of contributors increase, the unsustainability detected in the system increases. Due to the increase in part-time work experienced in the last 
economic crisis years, and since the approval of Law $1 / 2014$, a greater number of part-time workers will be covered by contributory retirement benefits because since this Law their conditions of access to retirement have been favoured. This fact causes a greater imbalance between the income from contributions and the pensions that will be received by workers, especially those that experience episodes of part-time work, of contributory unemployment and inactivity in their work careers.

It can be stated that future measures adopted to reduce the unemployment rate and increase fulltime employment will affect the viability of the pension system directly and therefore individual decisions related to retirement.

An added value of this research is including the probabilities of transition between employment states, and also incorporating the part-time state. It was considered necessary to include it due to an increase of this employment relationship in the last years, as well as the management of its pensions since Law 1/2014.

KEYWORDS: Viability, Part-time work, Unemployment, Survey of Living Conditions, Internal Rate of Return, IRR. 


\section{Introducción 1}

Los problemas de sostenibilidad a los que se tiene que enfrentar el sistema de pensiones español, provocado por el incremento del número de pensionistas sin el consiguiente relevo generacional, que permita mantener el equilibrio entre activos y pasivos, además del continuo aumento de la longevidad, provoca un desequilibrio creciente en el sistema de la Seguridad Social. Este hecho se ha agravado con la crisis económica, fundamentalmente por el descenso de los ingresos por cotizaciones y el incremento de los gastos por prestaciones contributivas. En este sentido, durante el periodo 2008-2015 los ingresos por cotizaciones experimentaron un descenso del 7,1\%, mientras que el gasto en pensiones contributivas se incrementó en un 36,5\%. Como consecuencia de esta evolución dispar, en el año 2015 las cotizaciones sociales solo cubrieron el 86,9\% del gasto en pensiones contributivas totales, mientras que en el año 2008 representaban el 127\%.

El sistema público de pensiones español es un sistema de reparto. Supone que las cotizaciones de los trabajadores se emplean para pagar las pensiones de los jubilados actuales, a cambio del compromiso de que las generaciones futuras paguen sus pensiones cuando éstos se jubilen, es decir, se trata de un sistema de transferencias intra e intergeneracional. Por ello, la sostenibilidad del sistema depende de la relación existente entre el número de cotizantes y el número de pensionistas. En este contexto, el incremento de la tasa de paro y el aumento de la longevidad tienden, por un lado, a reducir el número de cotizantes y por otro, a incrementar el número de pensiones, poniendo en peligro el equilibrio de tesorería, es decir, la relación entre los ingresos y los gastos del sistema. Por lo que, el análisis y la relación del sistema de pensiones con los cambios en el mercado de trabajo se hace imprescindible.

En el análisis del mercado de trabajo, y de forma más concreta de la tasa de desempleo, se debe tener en cuenta factores como la entrada y salida del mercado laboral, las transiciones desde la actividad a la inactividad laboral, la temporalidad, la parcialidad y la influencia de los distintos tipos de contratos en el empleo y en el paro, tal y como se puede comprobar en los trabajos de investigación de los últimos años. Cabe destacar, por ejemplo, los trabajos de Toharia (1998), que estudia los factores influyentes en el mercado de trabajo español; Cebrián, Moreno y Toharia (2009), quiénes analizan la entrada en el mercado de trabajo basándose en la Muestra Continua de Vidas Laborales; Malo y Toharia (1999a, 1999b), quiénes explican cuáles son los costes de despido y la creación de empleo en España; Malo y Toharia (2009), quiénes describen las posibles reformas del mercado de trabajo.

1.- Las autoras agradecen las sugerencias y comentarios de la investigadora Dra. $D^{a}$. Jennifer Alonso García (ARC Centre of Excellence in Population Ageing Research (CEPAR), Australia). 
Por su parte, Kugler, Jimeno y Hernanz (2002) analizan el empleo y las posibles reformas del mercado laboral español: Llorente, Sáez y Vera (2009) explican factores de la inserción laboral: y Rosado, Domínguez y Alonso (2015) introducen las transiciones laborales entre el empleo, el desempleo y la inactividad en el análisis de la solvencia financiera del sistema de pensiones público de jubilación.

En el análisis del incremento del paro y las diferencias respecto al resto de países de la Unión Europea, se deben considerar otras características específicas de nuestro mercado laboral, como es la elevada tasa de temporalidad, la propagación de las prejubilaciones que se han sucedido en los últimos años, y el incremento de los contratos a tiempo parcial, lo que ha propiciado que la población activa española tenga un perfil muy particular, que influye indirectamente en el crecimiento de la tasa de paro.

La preocupación por la salud financiera del sistema de la Seguridad Social en España es un hecho importante para todos los ciudadanos, ya que las pensiones públicas contributivas se han convertido en la única o principal fuente de ingreso de muchas familias. Por lo que no cabe duda que la sostenibilidad de las pensiones públicas ha sido y seguirá siendo una de las principales preocupaciones de la población y objeto de investigaciones para expertos e Instituciones.

En este sentido, y desde el año 2011 se han realizado importantes modificaciones sobre numerosos elementos del sistema de pensiones español. Casi todas las reformas y las investigaciones se han centrado en el sistema de jubilación y, en contadas ocasiones se ha analizado su solvencia, teniendo en consideración los retos a los que se enfrenta el sistema. La crisis económica ha provocado el aumento del desempleo y de la parcialidad laboral, elementos que deben considerarse en el análisis del sistema de pensiones de jubilación. Ello genera una interesante problemática desde el punto de vista social y económico, siendo necesario afrontar los cambios que se han producido en el mercado de trabajo y sobre las prestaciones más relevantes en España en cuanto a su número y cuantía, las pensiones contributivas de jubilación.

El objetivo de este trabajo es el estudio del efecto que el desempleo y la parcialidad laboral sobre la viabilidad financiera del sistema de pensiones de jubilación español. El análisis se desarrolla a partir de la Encuesta de Condiciones de Vida (ECV, 2011-2014) del INE, de la que se obtienen las probabilidades de transición entre el empleo a tiempo completo, el empleo a tiempo parcial, el desempleo contributivo y la inactividad, con las que se observan diferentes trayectorias laborales "tipo".

Tras esta introducción, la estructura del trabajo es la siguiente. En el epígrafe segundo se analizan las reformas del sistema de pensiones de jubilación español. En los epígrafes tercero y cuarto se detallan: la base de datos utilizada, el modelo utilizado en la estimación de las probabilidades de transición, la metodología empleada para medir la viabilidad del sistema y las hipótesis de trabajo. En el quinto epígrafe se presentan los resultados del Tanto Interno de Rendimiento probable ante diferentes trayectorias laborales. Por último, se recogen las conclusiones extraídas del trabajo y las referencias bibliográficas utilizadas. 


\section{Las reformas del sistema de pensiones de jubilación español}

El sistema público de pensiones de jubilación en España se basa en un sistema de reparto, en el que las pensiones a las que tiene que hacer frente el sistema cada año se pagan con las cotizaciones sociales de los trabajadores de ese mismo año, con la confianza de que ese proceso continuará en el futuro, esto es, que cuando ellos se jubilen recibirán una pensión pagada por los que estén en activo en ese momento (contrato intergeneracional). Además, se trata de un sistema de prestación definida porque la cuantía de la pensión queda fijada de antemano sobre la base del historial laboral de cada trabajador (salarios, años cotizados y edad de jubilación), es decir, que está relacionada, en mayor o menor medida, con el esfuerzo contributivo realizado a lo largo de la vida laboral.

El continuo envejecimiento demográfico y la crisis económica han anticipado en más de una década los déficits previstos en las cuentas de la Seguridad Social, y provocado que en los últimos años se hayan aprobado varias reformas cruciales para asegurar la sostenibilidad de nuestro sistema de pensiones.

De todas las medidas adoptadas, cabe destacar las introducidas por la Ley 27/2011, de 1 de agosto, sobre actualización, adecuación y modernización del sistema de Seguridad Social (en adelante, reforma de 2011), y la Ley 23/2013, de 23 de diciembre, reguladora del Factor de Sostenibilidad y del Índice de Revalorización del Sistema de Pensiones de la Seguridad Social (en adelante, reforma de 2013).

Entre las principales novedades que introdujo la reforma de 2011 cabe destacar las siguientes 2 :

- Retraso de la edad legal de jubilación hasta los 67 años. No obstante, la Ley 27/2011 mantiene la posibilidad de acceso a la jubilación desde los 65 años, con el 100\% de la pensión, cuando se tengan acumulados al menos 38,5 años de cotización. El objetivo de esta medida ha sido mejorar la relación entre el número de años cotizados y el número de años que se percibe la pensión de jubilación.

- Incremento hasta los 25 años el periodo de cálculo de la base reguladora. Siguiendo las reiteradas recomendaciones del Pacto Toledo, se intentó reforzar el principio de contributividad, estableciendo una mayor proporcionalidad entre el esfuerzo de cotización realizado a lo largo de la vida laboral y la pensión que se percibe a partir de la jubilación.

2.- La entrada en vigor de esta Ley tuvo lugar, con carácter general, el 1 de enero de 2013, si bien existe un periodo transitorio de 15 años para que su aplicación se haga de forma gradual y así suavizar su impacto. Esto supone que hasta el año 2027 la reforma no estará plenamente implantada. 
- La reforma de 2011 mantuvo en 15 años el periodo mínimo de cotización para generar el derecho a una pensión contributiva de jubilación, pero aumentó hasta 37 años el periodo de cotización necesario para alcanzar el 100\% de la base reguladora. Como consecuencia de esta modificación también se modificó la escala de los porcentajes aplicables para determinar la cuantía de la pensión que ahora adquiere una progresividad mensual, y es casi proporcional al número de meses cotizados a partir de los 15 primeros años.

- Se endurecen los requisitos necesarios para acceder a la jubilación anticipada con el objetivo de frenar el abandono temprano de la vida laboral y aproximar la edad real y la edad legal de jubilación. No obstante, a comienzos de 2013, tan solo unos meses después de la entrada en vigor de la reforma de 2011, el Gobierno aprobó el Real Decreto-ley 5/2013, de 15 de marzo, de medidas para favorecer la continuidad de la vida laboral de los trabajadores de mayor edad y promover el envejecimiento activo, que endureció aún más el acceso a la jubilación anticipada. Con carácter general, las posibilidades de jubilarse anticipadamente quedan del siguiente modo:

a) A una edad inferior en cuatro años a la edad legal de aplicación (67 o 65 años, según el caso) y con al menos 33 años cotizados siempre que el cese del trabajador sea por causa no imputable a él (jubilación anticipada involuntaria).

b) A una edad inferior en dos años a la edad legal de aplicación (67 o 65 años, según el caso) y con al menos 35 años cotizados, en el caso de jubilación voluntaria.

En ambos casos, a la pensión resultante se le aplica unos coeficientes reductores por cada trimestre que se anticipe la jubilación, siendo más elevados en el caso de la jubilación voluntaria. Estos porcentajes reductores dependen de los años que se tengan cotizados y el importe de la pensión resultante no puede superar el tope máximo de pensión reducido en un $0,50 \%$ por cada trimestre o fracción de anticipación.

- Se aumentan los incentivos a la prolongación de la vida laboral mejorando los porcentajes que se aplican por cada año que los individuos retrasen su jubilación. Así, a las personas que prolonguen su vida laboral más allá de su edad legal de jubilación, y siempre que al cumplir esta edad tengan cubierto el periodo mínimo de cotización, se les reconoce un porcentaje adicional por cada año completo cotizado entre su edad legal de jubilación y la edad a la que realmente se jubile. Este porcentaje depende del número de años cotizados que se acrediten en el momento en que se alcanza la edad legal de jubilación: un 2\% si tiene menos de 25 años cotizados; $2,75 \%$ si tiene entre 25 y 37 años cotizados; y $4 \%$ a partir de 37 años cotizados.

No cabe duda de que la Ley 27/2011, junto con las modificaciones introducidas por el Real Decretoley $5 / 2013$, introdujeron un conjunto de medidas de gran calado con el objetivo fundamental de preparar a nuestro sistema de pensiones frente al reto del envejecimiento demográfico. Sin embargo, al poco tiempo de aprobarse la Ley 27/2011 se publicaron diversos trabajos que afirmaban que ésta reforma era claramente incompleta e insuficiente pues no resolvía el problema de la sostenibilidad a largo de plazo de forma definitiva (De la Fuente y Doménech, 2013; Díaz Giménez y Díaz Saavedra, 2011; Conde-Ruiz y González, 2013 y Domínguez et al., 2011). Y es que, como señala Jiménez (2013), "el problema se puede resumir en una sola frase: nuestra generación es el doble que nuestros padres 
y nosotros somos el doble que nuestros hijos. Es decir, sostener las pensiones de nuestros mayores ha sido fácil hasta ahora, pero sostener las nuestras será prácticamente imposible, sobre todo si se pretende mantener el mismo nivel de cobertura y generosidad".

A finales del año 2012, a la amenaza estructural del envejecimiento demográfico se le unía una situación económica muy complicada pues la crisis había provocado la aparición de déficits en las cuentas de la Seguridad Social y además se preveía, como así está sucediendo, que esos déficits se intensificarían en los siguientes años dando lugar a mayores tensiones económicas en el corto plazo y a una fuerte reducción de los recursos acumulados en el Fondo de Reserva de la Seguridad Social ${ }^{3}$.

En este contexto, resultó imprescindible y urgente la adopción de nuevas medidas que contribuyeran a frenar el crecimiento del gasto en pensiones y, por ello, tan solo un año después de la entrada en vigor de la reforma 2011, se aprobó la Ley 23/2013 que introdujo nuevas medidas como son las siguientes:

En la reforma de 2011, siguiendo la línea de otros países europeos y las recomendaciones de la Comisión Europea, se introdujo por primera vez en nuestro sistema de pensiones el denominado factor de sostenibilidad aunque sin concretar las características del mismo, y retrasando su aplicación hasta el año 2027. Sin embargo, los problemas de sostenibilidad del sistema de pensiones español hacían aconsejable adelantar la aplicación del factor de sostenibilidad, como así se hizo con la reforma de 20134. La Ley 23/2013 define el factor como un instrumento que con carácter automático permite ajustar la cuantía de la pensión inicial de jubilación a la evolución de la esperanza de vida de acuerdo con la siguiente fórmula:

$$
F S_{t}=F S_{t-1} \cdot e^{*} 67
$$

Siendo $\mathrm{FS}_{\mathrm{t}}$ el factor de sostenibilidad del año t, $\mathrm{FS}_{\mathrm{t}-1}$ el factor de sostenibilidad del año t-1 y $e_{67}^{*}$ la variación interanual, en un periodo quinquenal, de la esperanza de vida a los 67 años según las tablas de mortalidad de la población pensionista de jubilación del sistema de la Seguridad Social.

3.- Desde finales de 2011, momento en el que el saldo del fondo alcanzó un máximo histórico de 66.815 millones de euros, las disposiciones realizadas han sido de 54.151 millones de euros. Esto ha supuesto que en tan solo cuatro años y medio su saldo se haya reducido en un 62\%, situándose a 30 de junio de 2016 en 25.176 millones de euros.

4.- Ya en el año 2011 diversos organismos y expertos como el Banco de España (2011) y De la Fuente y Doménech (2013) manifestaron que lo más sensato sería adelantar la introducción del Factor de Sostenibilidad al inicio de la reforma. En este mismo sentido, el Consejo Europeo aprobó el 30 de mayo de 2012 una Recomendación sobre el Programa Nacional de Reformas 2012 de España, en el que se instaba al Gobierno a acelerar la introducción del Factor de Sostenibilidad. Siguiendo estas recomendaciones, la Ley Orgánica 2/2012, de 27 de abril, de Estabilidad Presupuestaria y Sostenibilidad Financiera facultó al Gobierno para adelantar la aplicación del factor de sostenibilidad en caso de proyectar un déficit en el largo plazo en el sistema de pensiones. 
El factor de sostenibilidad se aplicará anualmente a partir de 2019 y su valor se revisará cada cinco años aplicando la siguiente fórmula:

$$
e_{67}^{*}=\left[\frac{e_{67}^{t-7}}{e_{67}^{t-2}}\right]^{1 / 5}
$$

Por ejemplo, la variación interanual del año 2019 y que se aplicará durante el periodo 2019-2023 será:

$$
e_{67}^{2019-2023}=\left(\frac{e_{67}^{2012}}{e_{67}^{2017}}\right)^{\frac{1}{5}}
$$

La variación interanual del año 2024 y que se aplicará en el periodo 2024-2028 será:

$$
e_{67}^{2024-2028}=\left(\frac{e_{67}^{2017}}{e_{67}^{2022}}\right)^{\frac{1}{5}}
$$

Y así sucesivamente.

Conviene resaltar que la implantación del Factor de Sostenibilidad transforma, al menos parcialmente, el actual sistema de reparto y prestación definida en uno de reparto y contribución definida, ya que la cuantía de la pensión de jubilación deja de depender únicamente del historial laboral del trabajador y estará en función también de la esperanza de vida en el momento de la jubilación. Además, modifica las "reglas del juego" asegurando al sistema frente al riesgo asociado al incremento de la longevidad. A partir de 2019, serán los nuevos jubilados los que deberán soportar los efectos del aumento de la esperanza de vida con una disminución en la cuantía de su pensión inicial o, si lo prefieren, con un retraso en la edad de jubilación que compense esa disminución.

Según Vidal (2014), la introducción del Factor de Sostenibilidad es una forma de introducir en el sistema público de pensiones de España alguno de los principios que rigen el denominado sistema de cuentas nocionales, aunque de una forma poco transparente y con lagunas técnicas.

Además del Factor de Sostenibilidad, la reforma de 2013 también introdujo otra medida importante como es el Índice de Revalorización de las Pensiones (IRP). Durante décadas la revalorización de las pensiones en España se ha efectuado en función del IPC con el objetivo de garantizar el poder adquisitivo de las pensiones. Sin embargo, el nuevo IRP supone un cambio radical en esta política de revalorizaciones al desligar la actualización anual de las pensiones del IPC y vincularla al necesario equilibrio presupuestario del sistema para que este sea sostenible, relegando, por tanto, a un segundo plano el mantenimiento del poder adquisitivo de los pensionistas. 
Según las nuevas reglas, que entraron en vigor el 1 de enero de 2014, las pensiones contributivas se revalorizarán en función de una serie de variables relacionadas con la situación financiera de la Seguridad Social, como son los ingresos y gastos del sistema, el número de pensiones y el efecto sustitución 5 .

En concreto, el valor del IRP de cada año se determina a partir de la siguiente fórmula:

$$
I R_{t+1}=\bar{g}_{I, t+1}-\bar{g}_{P, t+1}-\bar{g}_{s, t+1}+\alpha\left(\frac{I_{t+1}^{*}-G_{t+1}^{*}}{G_{t+1}^{*}}\right)
$$

Siendo:

$I R_{t+1}$ : Índice de revalorización de pensiones del año $t+1$ (año para el que se calcula la revalorización) expresado en tanto por uno con cuatro decimales.

$\bar{g}_{l, t+1}$ : Media móvil aritmética, centrada en $t+1$, de once valores de la tasa de variación de los ingresos de la Seguridad Social.

$\bar{g}_{P, t+1}:$ Media móvil aritmética, centrada en $t+1$, de once valores de la tasa de variación del número de pensiones contributivas de la Seguridad Social.

$\bar{g}_{s, t+1}$ : Media móvil aritmética, centrada en $\mathrm{t}+1$, de once valores del efecto sustitución.

$I^{*} t+1$ : Media móvil geométrica, centrada en $t+1$, de once valores del importe de los ingresos de la Seguridad Social.

$G_{t+1}^{*}$ : Media móvil geométrica, centrada en $\mathrm{t}+1$, de once valores del importe de los gastos de la Seguridad Social.

$\alpha$ : $\quad$ Parámetro que determina la velocidad con la que se van a corregir los desequilibrios presupuestarios del sistema. La ley 23/2013 establece que este parámetro debe oscilar entre un valor mínimo del 0,25 y un máximo del 0,33. El valor de este parámetro se revisará cada cinco años, fijándose para el quinquenio 2014-2019 en el 0,25.

Es importante destacar que para evitar cambios excesivamente bruscos, así como posibles reducciones en términos nominales de la pensión, el IRP incluye una cláusula suelo para que todos los años las pensiones crezcan un mínimo garantizado $(0,25 \%)$ y un techo que es igual a la variación del IPC en el periodo anual anterior más $0,5 \%$ (IPC+0,5\%).

5.- La Ley 23/2013 define el efecto sustitución como "la variación interanual de la pensión media del sistema en un año en ausencia de revalorización en dicho año". Esto supone que el efecto sustitución refleja sobre todo la repercusión que sobre la cuantía de la pensión media tiene el hecho de que las nuevas pensiones que entran en el sistema a lo largo de un año son de mayor cuantía que las que causan baja en ese mismo año, pero también recoge el efecto sobre la pensión media derivada de otras circunstancias como el aumento del complemento a mínimo al cumplir 65 años, los cambios de grado en las pensiones de incapacidad permanente, los cambios normativos, etc. 
Es evidente, que el objetivo fundamental del IRP no es criticable: lograr la convergencia entre ingresos y gastos del sistema, esto es, que no se reparta más de lo que se tiene. Sin embargo, esta medida ha sido muy criticada por el sacrificio que va a imponer a los pensionistas, ya que serán estos, vía pérdida de poder adquisitivo, los que tendrán que soportar el ajuste necesario para alcanzar el equilibrio presupuestario 6 . En efecto, a diferencia del resto de medidas aprobadas, cuyo coste es soportado exclusivamente por los futuros pensionistas, el IRP afectará también a los actuales pensionistas.

Por otra parte, y según AIREF (2015) y Roch et al. (2017) el cálculo del IRP presenta un problema de circularidad, ya que para obtener el IRP aplicable a las pensiones en un determinado año, la fórmula implica conocer, entre otras variables, la proyección del gasto total en pensiones que al mismo tiempo, depende del IRP.

Son muchos los autores que han estudiado los efectos de la Ley $23 / 2013$, entre los que destacan: Herce (2013), Conde-Ruiz (2013), Meneu et al. (2013), Devesa et al. (2013 a,b,c), Devesa et al. (2014), Rosado y Domínguez (2014), Hoyo (2014) y Sánchez (2014). Todos ellos coinciden en que se producirá una mejora de la solvencia del sistema de pensiones español en detrimento del poder adquisitivo de los pensionistas, tanto actuales como futuros. Estos autores, si bien han abordado sus trabajos desde diversas ópticas, no han incluido el efecto de la parcialidad en sus análisis, lo que se convierte en el objetivo de este trabajo. Para ello, se tendrá en cuenta en el cálculo de las pensiones de estos trabajadores, según las reglas y modificaciones efectuadas a partir de la Ley 1/2014, de 28 de febrero, para la protección de trabajadores a tiempo parcial.

Esta nueva reforma modifica el cálculo de los periodos de cotización para acceder a la pensión de jubilación, respecto de los periodos acreditados con contratos de tiempo parcial, incluidos los contratos de trabajo fijo discontinuo a tiempo parcial, con independencia de que la reducción de la jornada se realice en cómputo diario, semanal, mensual 0 anual.

A pesar de que el Real Decreto Ley 11/2013, de 2 de agosto, para la protección de trabajadores a tiempo parcial y otras medidas urgentes en el orden económico y social, había flexibilizado el número de años requeridos para acceder a la jubilación, la Ley 1/2014 recoge la fórmula para exigir el mismo esfuerzo a un trabajador a tiempo completo que a un trabajador a tiempo parcial, evitando que se produzcan efectos desproporcionados entre las cotizaciones realmente efectuadas por el trabajador y la cuantía de la pensión de jubilación.

6.- Por ello, la Disposición Adicional Tercera de la Ley 23/2013 establece que el Gobierno deberá elaborar cada cinco años un estudio sobre los efectos de la reforma en el poder adquisitivo de los pensionistas, que será revisado en el marco del diálogo social y en el Congreso de los Diputados. 
En este sentido, en el cálculo de la primera pensión de jubilación se aplican las siguientes reglas:

- Se tienen en cuenta los distintos periodos durante los cuales el trabajador haya permanecido en un contrato a tiempo parcial. A tal efecto, se aplica el coeficiente de parcialidad ${ }^{7}$ sobre el periodo de alta en el contrato, siendo su resultado el número de días que se consideran efectivamente cotizados.

- Una vez determinados el número de días totales cotizados, se calcula el coeficiente global de parcialidad8.

- En el cálculo de la pensión de jubilación, el número de días cotizados que resulten se incrementa con la aplicación del coeficiente 1,5 sin que el número de días resultante pueda ser superior al periodo de alta a tiempo parcial.

Con el objetivo de eliminar 0 , al menos, disminuir la brecha de género en pensiones, en el año 2015 se aprobó el complemento para las pensiones de mujeres con hijos naturales 0 adoptados y sean beneficiarias en cualquier régimen de Seguridad Social de pensiones contributivas de jubilación, viudedad e incapacidad permanente. Dicho complemento tiene a todos los efectos naturaleza jurídica de pensión pública contributiva, y consiste en un importe equivalente al resultado de aplicar a la cuantía inicial de las referidas pensiones un porcentaje determinado, que se encuentra en función del número de hijos (por dos hijos será del $5 \%$; subirá al $10 \%$ cuando sean tres hijos y con cuatro o más, alcanzará el 15\%).

A partir del Real Decreto-ley 3/2016, de 2 diciembre, por el que se adoptan medidas en el ámbito tributario dirigidas a la consolidación de las finanzas públicas y otras medidas urgentes en materia social, se han incrementado los topes máximos futuros y bases máximas de cotización, así como del límite máximo de las pensiones del sistema de Seguridad Social.

En la actualidad, son diversas las medidas de reforma que se plantean entre la opinión pública y expertos, con el objetivo de mejorar la sostenibilidad del sistema público de pensiones. Entre las medidas que se proponen destacan las siguientes: elevar la edad de jubilación, utilizar toda la vida laboral para el cálculo de la pensión, incrementar el número de años para obtener el 100\% de la pensión de jubilación, avanzar en la implantación de un sistema de cuentas individuales, destopar las cotizaciones y eliminar las bonificaciones, financiar las pensiones de viudedad y orfandad con impuestos, el diseño de un complemento en el IRPF 0 aumentar un punto el impuesto, aumentar un punto el IVA, incrementar el ahorro para complementar la pensión y realizar reformas estructurales en el mercado de trabajo.

7.- El coeficiente de parcialidad se determina por el porcentaje de la jornada realizada a tiempo parcial respecto de la jornada realizada por un trabajador a tiempo completo comparable.

8. - El coeficiente global de parcialidad es el porcentaje que representa el número de días cotizados acreditados sobre el total de días en alta a lo largo de toda la vida laboral del trabajador. 


\section{3.- La base de datos: la Encuesta de Condiciones de Vida (2011-2014)}

La finalidad I de la Encuesta de Condiciones de Vida (en adelante, ECV) es la producción sistemática de estadísticas comunitarias sobre la renta y las condiciones de vida, que incluyan datos transversales y longitudinales comparables y actualizados sobre la renta, el nivel y composición de la pobreza y la exclusión social, a escala nacional y europea.

Aunque lo prioritario en la ECV es la producción de información transversal con un alto grado de calidad en lo que a su actualidad y comparabilidad se refiere, su diseño permite también obtener información longitudinal, es decir, referida a las mismas personas en diferentes momentos a lo largo del tiempo. De esta forma, se podrá conocer el cambio bruto, es decir, la evolución temporal de las variables de interés en cada individuo, y el estudio de transiciones, duraciones e intervalos entre sucesos. En concreto, para este trabajo se utilizan los datos longitudinales correspondientes al periodo 2011-2014.

Según Cabezas (2012), una de las ventajas que ofrece la disponibilidad de datos longitudinales es la posibilidad de estudiar transiciones laborales. De esta forma, se observan las transiciones entre la inactividad, el desempleo contributivo, el empleo a tiempo parcial y el empleo a tiempo completo. En nuestro análisis se proyectan las trayectorias laborales futuras a partir de trayectorias tipos para individuos con determinadas características, con el objetivo de construir historias laborales completas a partir de las cuáles se obtienen sus pensiones de jubilación correspondientes en base a las reformas de 2011 y 2013.

Para llevar a cabo este trabajo se ha optado por la utilización de la Encuesta de Condiciones de Vida (ECV) del INE, en su versión más actual para realizar análisis longitudinales (2011-2014), con la finalidad de obtener transiciones laborales entre los estados de una forma más desagregada (por edad, por género, por tipo de contrato, por jornada laboral...). Y es por ello que se prescinde de la utilización de otras fuentes de información alternativas como puede ser la Encuesta de Población Activa del INE, en la que se analizan las transiciones laborales de la población empleada, parada e inactiva pero de una forma más genérica, ya que nuestro objetivo primordial es analizar cómo influye la parcialidad y el desempleo en la viabilidad del sistema de pensiones de jubilación español, y factores como son la edad y el género determinan las transiciones hacia estos estados laborales.

A pesar de que puede resultar criticable la no utilización de la población cotizante en nuestro análisis y hacerlo a partir de los individuos que conforman la ECV, se ha priorizado la posibilidad de obtención de las probabilidad de transición desde los contratos a tiempo completo y parciales, y el poder aplicarlas a sus carreras laborales futuras. 
Por otra parte, y debido a la facilidad del tratamiento de la información, se ha preferido utilizar la ECV en lugar de la Muestra Continua de Vidas Laborales (MCVL) del Ministerio de Empleo y Seguridad Social, dejando para investigaciones futuras la comparación de los resultados con ambas bases de datos.

A partir de la ECV, se ha seleccionado una muestra de individuos entre los 25 y los 66 años de edad que durante el periodo (2011-2014) hayan tenido una de las situaciones laborales anteriormente indicadas, y se ha comparado su situación al final del periodo indicado con la inicial, con el objeto de comprobar la evolución del empleo y de forma más concreta, del empleo a tiempo parcial.

Considerando la parcialidad de los contratos, se ha realizado un análisis descriptivo de la ECV (2011-2014). De esta forma, el Cuadro 1 recoge la incidencia del empleo a tiempo parcial, obteniendo por un lado la proporción de empleo a tiempo parcial sobre el total de la Encuesta, que incluye tanto a los empleados, a los desempleados contributivos como a los inactivos, y por otro lado, la proporción de empleo a tiempo parcial sobre el total de los empleados. A partir de los resultados expuestos, se puede comprobar el incremento significativo del empleo parcial respecto al total de la encuesta y al empleo entre los años 2011 y 2014. En cuanto, a la evolución del empleo parcial por género, se observa como éste se ha incrementado en los últimos años tanto para hombres y mujeres. No obstante, la incidencia del empleo a tiempo parcial sigue siendo muy superior en las mujeres: en 2014 una de cada cuatro mujeres empleadas tenían un trabajo a tiempo parcial, lo que suponía el $70 \%$ del empleo parcial total.

\section{Cuadro 1. Evolución del empleo a tiempo parcial (2011- 2014)}

\begin{tabular}{|l|cc|cc|cc|}
\hline Años & \multicolumn{2}{|c|}{ Ambos sexos } & \multicolumn{2}{c|}{ Hombres } & \multicolumn{2}{c|}{ Mujeres } \\
& Parcial/Total & Parcial/Empleo & Parcial/Total & Parcial/Empleo & Parcial/Total & Parcial/Empleo \\
\hline $\mathbf{2 0 1 1}$ & $6,43 \%$ & $13,56 \%$ & $3,17 \%$ & $5,95 \%$ & $9,56 \%$ & $22,91 \%$ \\
$\mathbf{2 0 1 2}$ & $6,58 \%$ & $14,49 \%$ & $3,34 \%$ & $6,59 \%$ & $9,69 \%$ & $23,95 \%$ \\
$\mathbf{2 0 1 3}$ & $7,01 \%$ & $15,80 \%$ & $3,87 \%$ & $7,83 \%$ & $10,00 \%$ & $25,28 \%$ \\
$\mathbf{2 0 1 4}$ & $7,16 \%$ & $15,91 \%$ & $3,93 \%$ & $7,81 \%$ & $10,24 \%$ & $25,58 \%$ \\
\hline
\end{tabular}

FUENTE: Elaboración propia a partir de la Encuesta de Condiciones de Vida (2011-2014).

En el Gráfico 1, se realiza una comparativa de la situación laboral en España durante el periodo analizado respecto a la situación laboral de los individuos que conforman la ECV (2011-2014) de forma global, tanto para hombres y mujeres. De esta forma, y como se puede observar el porcentaje de población en empleo a tiempo completo ha descendido en el periodo 2011-2014, sin embargo, el empleo a tiempo parcial y el desempleo contributivo se ha incrementado a lo largo de los años. 
Por otra parte, el porcentaje de población inactiva es importante, ya que en nuestro análisis se considera en esta categoría a todas las personas que no están empleadas ni perciben prestaciones por desempleo contributivo (personas que se ocupan de su hogar, estudiantes, jubilados y prejubilados personas que perciben una pensión distinta de la jubilación y prejubilación, los incapacitados para trabajar y las personas que, sin ejercer ninguna actividad económica, reciben ayudas públicas o privadas).

En cuanto a la comparativa respecto al género, se puede comprobar, como se ha comentado anteriormente, que la parcialidad es superior en el caso de las mujeres en todos los años analizados, y lo mismo sucede en cuanto a la inactividad. A partir de lo cual, se concluye que son las mujeres, debido a diferentes circunstancias laborales y familiares, las que cotizan en menor medida a nuestro sistema de la Seguridad Social.

\section{Gráfico 1. Situación Laboral en España por sexo (2011. 2014)}

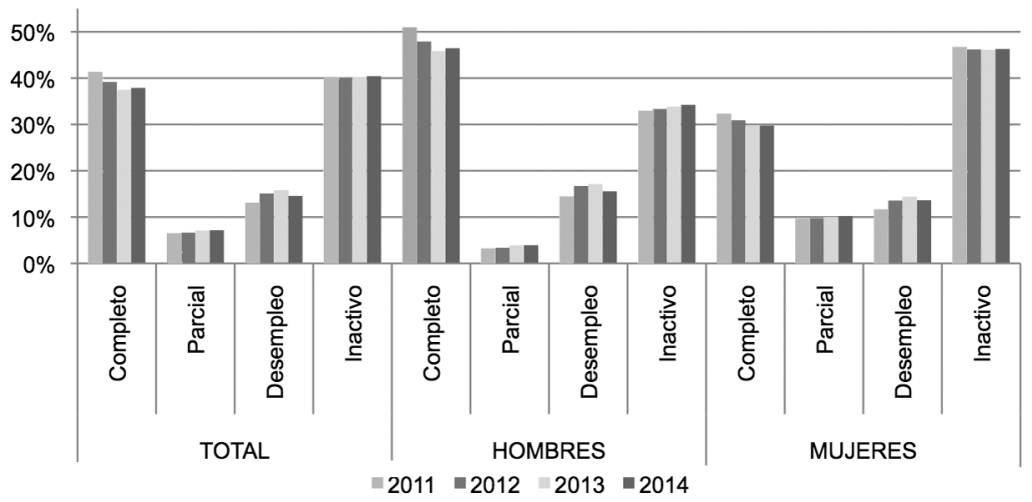

FUENTE: Elaboración propia a partir de la Encuesta de Condiciones de Vida (2011-2014).

En cuanto al análisis realizado por tramos de edad (Gráfico 2), se observa cómo a medida que se incrementa la edad de los individuos que conforman la ECV en el año 2014, aumenta el empleo a tiempo completo y a tiempo parcial, siendo los tramos de edad entre los 35 y los 44 años, los que presenta un mayor porcentaje de individuos con empleos a tiempo completo y parcial respecto al total. Tal es así, que la parcialidad destaca entre los más jóvenes, en los tramos de edad entre 20-24 y 25-29 años de edad. Sin embargo, los individuos entre 16 y 19 años de edad presentan un elevado porcentaje de inactividad, al igual que a partir del tramo 60-64 años debido a que una parte importante de la población pasa a ser pensionista. 


\section{Gráfico 2. Situación Laboral en España en 2014 por tramos de edad}

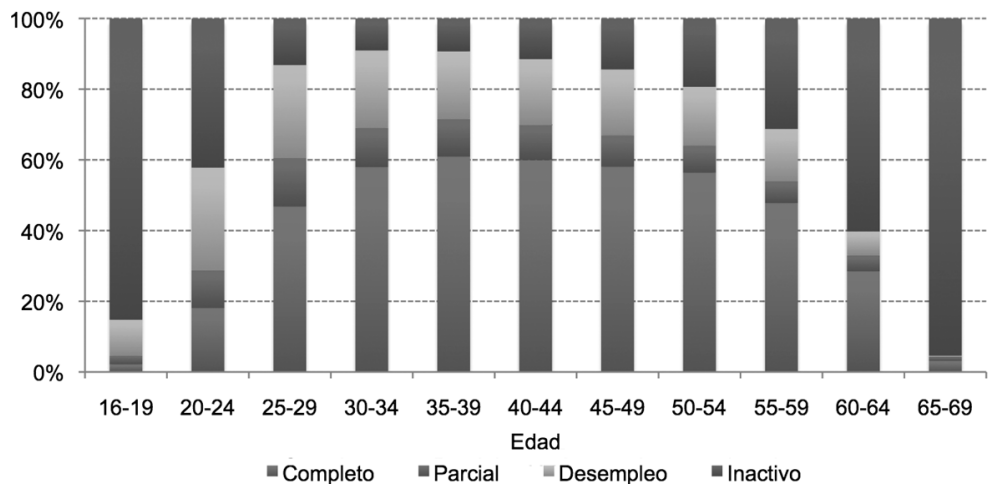

FUENTE: Elaboración propia a partir de la Encuesta de Condiciones de Vida (2011-2014).

\section{4.- Análisis empírico}

\subsection{Estimaciones de las probabilidades de transición}

En nuestro análisis se obtiene el TIR para un individuo "tipo" que suponemos accede al mercado laboral a la edad de 25 años y se jubila a los 67 años (edad legal), para el cual vamos a suponer diferentes escenarios del mercado de trabajo, que se van a obtener a partir de las probabilidades de transición entre el Empleo a Tiempo Completo (TC), a Tiempo Parcial (TP), el Desempleo Contributivo (D) y la Inactividad (I) extraídas a partir de la Encuesta de Condiciones de Vida (2011-2014), con el objetivo de realizar la comparativa sobre la evolución de las transiciones laborales y de forma más concreta, sobre el incremento de la parcialidad en los contratos.

Siguiendo a Alonso y Rosado (2016), en el cálculo de las transiciones laborales, se ha considerado las siguientes situaciones laborales en las que puede estar el individuo: Empleado a Tiempo Completo ( $j=E C)$; Empleado a Tiempo Parcial ( $j=E P)$; Desempleado (j=D); o Inactivo (j=I). 
Adoptamos un proceso estocástico ${ }^{9}$ de tiempo discreto $\left\{Y \_t, t=0,1,2,3, \ldots\right\}$ que representa el estado donde los individuos son en el periodo de referencia. Asumimos, así que $Y_{t}$ es un proceso de Markov, que implica que la probabilidad de un individuo $i$ en estado $J_{t}$ en el momento $t$, sólo depende de la información disponible más actual y es independiente de su estado anterior. Matemáticamente, se representa como sigue:

$$
\operatorname{Pr}\left(Y_{t}^{i}=j_{t} \mid Y_{0}^{i}=j_{0}, Y_{1}^{i}=j_{1}, \ldots, Y_{t-2}^{i}=j_{t-2}, Y_{t-1}^{i}=j_{t-1}\right)=\operatorname{Pr}\left(Y_{t}^{i}=j \mid Y_{t-1}^{i}=j_{t-1}\right)=P_{j_{t-1} j_{t}}^{i}
$$

La siguiente matriz $T^{i}$ muestra las diferentes probabilidades de transición para un individuo iy para los estados laborales $j$ y $k$, y que se denota como $P{ }_{j k}$ para un periodo determinado:

$$
T^{i}=\left(\begin{array}{cccc}
P_{E C E C}^{i} & P_{E C E P}^{i} & P_{E C D}^{i} & P_{E C I}^{i} \\
P_{E P E C}^{i} & P_{E P E P}^{i} & P_{E P D}^{i} & P_{E P I}^{i} \\
P_{D E C}^{i} & P_{D E P}^{i} & P_{D D}^{i} & P_{D I}^{i} \\
P_{I E C}^{i} & P_{I E P}^{i} & & P_{I I}^{i}
\end{array}\right)
$$

Los componentes de la matriz de transición T representan las probabilidades de transición entre Ios estados laborales. Las probabilidades $P i_{j j}$, para $j=E C, E P, D, I$ representan las probabilidades de permanencia, es decir, la probabilidad de que un individuo permanece en el estado $j$ durante el período estudiado, mientras que $P i_{j k}$, para $j \neq k$ donde $j, k=E C, E P, D, I$ representa la probabilidad de una transición de estado $j$ al estado $k$ durante el período estudiado (Marston et al., 1976).

Se ha estimado un modelo de probabilidades de transición entre los estados laborales a parttir de un modelo logit multinominal, tal y como se muestra en la siguiente expresión:

$$
P_{j k}^{i, x}=\operatorname{Pr}\left(Y_{i t}^{x}=k \mid Y_{i t-1}^{x-1}=j\right)=\frac{\exp \left(\beta_{j} Z_{i j}\right)}{\sum_{l} \exp \left(\beta_{l} Z_{i j}\right)}, j, k=E, U, I,
$$

Donde $i$ representa al individuo y $Z_{i j}$ las características individuales que incluye el estado laboral actual $j$.

A partir de los datos de la ECV se ha obtenido la Muestra que se recoge en el Cuadro 2 a la que se le han aplicado los filtros siguientes: 


\section{Cuadro 2. Enlace y filtros aplicados a la población de la ECV}

\begin{tabular}{|l|c|}
\hline Número de Individuos de la Muestra (2013-2014) & \\
\hline Enlace de los individuos de la ECV en el año 2013 que se mantienen en el año 2014. & 12.737 \\
Se han eliminado a aquellos individuos mayores de 66 años de edad & 9.926 \\
Se han eliminado a aquellos individuos con pensiones de jubilación u otro tipo de pensiones contributivas, como las de incapacidad & 9.595 \\
\hline
\end{tabular}

FUENTE: Elaboración propia a partir de la Encuesta de Condiciones de Vida (2011-2014).

La disponibilidad de datos longitudinales nos permite analizar las transiciones laborales entre los diferentes estados, considerando las situaciones de empleo a tiempo completo, empleo a tiempo parcial, desempleo contributivo e inactividad, tal y como se muestran en los Cuadros 3 y 4 de forma genera, l y desagregada por tramos de edad.

Los resultados ponen de manifiesto las probabilidades de permanencia y de transición entre los estados laborales. De esta forma, se observa que la probabilidad de permanecer en un empleo a tiempo parcial es del $54,38 \%$, frente a prácticamente el $78 \%$ de mantenerse en el empleo a tiempo completo.

En cuanto al análisis desagregado en función de la edad de los individuos (Cuadro 4), se observa cómo en las edades más jóvenes la transición hacia el desempleo es más elevada, siendo la probabilidad de mantenerse en el empleo a tiempo completo más baja respecto a los tramos de edad intermedios, entre los 25 y los 44 años de edad. Sin embargo, la probabilidad de transición, de aquellos individuos entre los 55 y los 64 años, edades cercanas a la jubilación, hacia el desempleo y hacia la inactividad es superior respecto a las edades intermedias, suponiendo una probabilidad de mantenerse en el desempleo de $64 \%$ y del $88,61 \%$ en la inactividad.

\section{Cuadro 3. Probabilidades de transición entre los estados laborales y tipo de jornada en España en 2013 y 2014}

\begin{tabular}{|l|ccccc|}
\hline \multirow{2}{*}{ Estado Laboral en t } & \multicolumn{4}{|c|}{ Estado Laboral en t+n } \\
\cline { 2 - 6 } & Empleo TC & Empleo TP & Desempleo Contributivo & Inactivo & Total \\
\hline Empleo TC & $77,95 \%$ & $6,95 \%$ & $12,68 \%$ & $2,42 \%$ & $100,00 \%$ \\
Empleo TP & $40,12 \%$ & $54,38 \%$ & $4,14 \%$ & $1,36 \%$ & $100,00 \%$ \\
Desempleo Contributivo & $13,60 \%$ & $13,45 \%$ & $63,00 \%$ & $9,95 \%$ & $100,00 \%$ \\
Inactivo & $8,28 \%$ & $7,65 \%$ & $0,00 \%$ & $84,07 \%$ & $100,00 \%$ \\
\hline
\end{tabular}

FUENTE: Elaboración propia a partir de la Encuesta de Condiciones de Vida (2011-2014). 


\section{Cuadro 4. Probabilidades de transición entre los estados laborales y tipo de jornada en España en 2013 y 2014 por tramos de edad}

\begin{tabular}{|c|c|c|c|c|c|c|}
\hline \multicolumn{2}{|c|}{ Estado Laboral en $\mathrm{t}$} & \multirow{2}{*}{\multicolumn{5}{|c|}{ Estado Laboral en $t+n$}} \\
\hline & & & & & & \\
\hline & & \multirow{2}{*}{$\begin{array}{c}\text { Empleo TC } \\
75,78 \%\end{array}$} & \multirow{2}{*}{$\begin{array}{c}\text { Empleo TP } \\
6,28 \%\end{array}$} & \multirow{2}{*}{$\begin{array}{c}\begin{array}{c}\text { Desempleo } \\
\text { Contributivo }\end{array} \\
14,90 \%\end{array}$} & \multirow{2}{*}{$\begin{array}{c}\text { Inactivo } \\
3,05 \% \\
\end{array}$} & \multirow{2}{*}{$\begin{array}{c}\text { Total } \\
100,00 \% \\
\end{array}$} \\
\hline De 25 a 34 años & Empleo TC & & & & & \\
\hline & Empleo TP & $43,19 \%$ & $51,00 \%$ & $3,29 \%$ & $2,56 \%$ & $100,00 \%$ \\
\hline & Desempleo Contributivo & $11,00 \%$ & $16,67 \%$ & $63,50 \%$ & $8,90 \%$ & $100,00 \%$ \\
\hline & Inactivo & $9,70 \%$ & $7,33 \%$ & $0,00 \%$ & $83,00 \%$ & $100,00 \%$ \\
\hline \multirow[t]{4}{*}{ De 35 a 44 años } & Empleo TC & $71,00 \%$ & $8,27 \%$ & $18,10 \%$ & $2,60 \%$ & $100,00 \%$ \\
\hline & Empleo TP & $42,00 \%$ & $50,00 \%$ & $6,41 \%$ & $1,64 \%$ & $100,00 \%$ \\
\hline & Desempleo Contributivo & $15,00 \%$ & $16,50 \%$ & $59,00 \%$ & $9,50 \%$ & $100,00 \%$ \\
\hline & Inactivo & $10,58 \%$ & $5,59 \%$ & $0,00 \%$ & $83,83 \%$ & $100,00 \%$ \\
\hline \multirow[t]{4}{*}{ De 45 a 54 años } & Empleo TC & $81,46 \%$ & $5,15 \%$ & $11,39 \%$ & $2,00 \%$ & $100,00 \%$ \\
\hline & Empleo TP & $41,00 \%$ & $50,14 \%$ & $7,62 \%$ & $1,24 \%$ & $100,00 \%$ \\
\hline & Desempleo Contributivo & $10,00 \%$ & $10,90 \%$ & $65,83 \%$ & $13,27 \%$ & $100,00 \%$ \\
\hline & Inactivo & $7,39 \%$ & $6,96 \%$ & $0,00 \%$ & $85,65 \%$ & $100,00 \%$ \\
\hline \multirow[t]{4}{*}{ De 55 a 64 años } & Empleo TC & $78,90 \%$ & $5,20 \%$ & $12,18 \%$ & $3,72 \%$ & $100,00 \%$ \\
\hline & Empleo TP & $42,17 \%$ & $48,00 \%$ & $6,53 \%$ & $3,30 \%$ & $100,00 \%$ \\
\hline & Desempleo Contributivo & $9,50 \%$ & $10,00 \%$ & $64,00 \%$ & $16,50 \%$ & $100,00 \%$ \\
\hline & Inactivo & $5,50 \%$ & $5,89 \%$ & $0,00 \%$ & $88,61 \%$ & $100,00 \%$ \\
\hline
\end{tabular}

FUENTE: Elaboración propia a partir de la Encuesta de Condiciones de Vida (2011-2014).

\subsection{Metodología: El Tanto Interno de Rendimiento (TIR)}

Para medir la sostenibilidad de un sistema de pensiones existen diversos indicadores actuariales pero, sin duda, el más utilizado es el denominado Tanto Interno de Rendimiento (TIR). Siguiendo a Devesa, Lejárraga y Vidal (2002), matemáticamente el TIR es el tipo de interés real de la ley de capitalización compuesta que iguala el valor actuarial de las cotizaciones con el valor actuarial de las pensiones.

En este trabajo se ha optado por este método para analizar el equilibrio financiero de individuos "tipo" o cotizantes representativos, a pesar de los inconvenientes que enumeran Devesa, Lejárraga y Vidal (2002), en cuanto a la falta de claridad sobre los elementos que inciden en el tanto al convertirlo en representativo del sistema. Este mismo enfoque lo utilizan Clingman et al. (2014), Leimer (1994) y OSFI (2012), en cuanto al análisis de la equidad del sistema bajo supuestos de un cotizante o grupo de cotizantes. 
La relación entre el TIR y la sostenibilidad de un sistema de reparto tiene su origen en la proposición de Samuelson (1958). La idea básica de Samuelson es que un sistema de pensiones basado en el reparto es financieramente sostenible a largo plazo si su TIR no supera la tasa de crecimiento real del salario medio más la tasa de crecimiento de la población cotizante 0 , lo que es lo mismo, no supera el crecimiento de la base fiscal del sistema que, en el largo plazo, es igual a la tasa de crecimiento del PIB real.

Por su parte, Murphy y Welch (1998) afirman que la viabilidad del sistema de pensiones de reparto dependerá del crecimiento económico sostenible promedio a largo plazo. Y tal y como señalan Jimeno y Licandro (1999), dado que en el largo plazo la tasa de crecimiento de los salarios reales viene dada por la tasa de crecimiento de la productividad del trabajo, el sistema de pensiones será sostenible siempre que su TIR, es decir, que la rentabilidad que proporciona a sus participantes, no supere la tasa media de crecimiento del PIB real a largo plazo, que es el límite marcado por Samuelson.

Esta metodología también es utilizada por diversos autores para estudiar la equidad y equilibrio de los sistemas de pensiones, entre los que podemos destacar a: Jimeno (2003), Domínguez y Encinas (2008), Devesa y Devesa (2010), Devesa et al. (2011), Alonso y Pazos (2010), Blasco (2013), Rosado y Domínguez (2014), Rosado, Domínguez y Alonso (2015).

La fórmula que nos permite calcular el valor actuarial de las cotizaciones de un trabajador a lo largo de su período activo, se calcula mediante la siguiente ecuación:

$$
V_{c o t}=\sum_{t=0}^{x_{j}-1-x_{e}} P_{x_{e}}^{t} C \quad W_{x_{e}} \prod_{i=t}^{x_{j}-1-x_{e}}(1+\alpha)^{t}(1+\beta)^{-t}(1+i)^{-t}
$$

Donde la notación utilizada es:

$\mathrm{i}=$ Tanto Interno de Rendimiento real.

$x_{e}=$ Edad del individuo al incorporarse al mercado laboral.

$x_{j}=$ Edad del individuo al alcanzar la jubilación.

$P_{x e}^{t}=$ Probabilidad de que una persona de edad , - .años alcance la edad, - .+ años.

$C=$ Porcentaje de cotización. Comprende tanto la aportación del empresario como la del trabajador. Se supone constante.

$W_{x e}=$ Salario a la edad $x_{e}$, que se supone coincidente con la base de cotización.

$\alpha=$ Tanto anual de crecimiento nominal de los salarios, que se supone constante.

$\beta=$ Tanto anual de crecimiento de la inflación, que se supone constante. 
El valor actual actuarial de las prestaciones por jubilación, descontadas al tanto i y aplicando de manera simplificada la legislación española en vigor, es:

$$
V_{R X}=\sum_{t=X_{j}-X_{e}}^{W-1-X_{e}} R_{X_{j}} P_{X_{e}}^{t}\left(1+\lambda^{*}\right)^{t-\left(X_{j}-X_{e}\right)}(1+\beta)^{-t}(1+i)^{-t}
$$

Donde la notación utilizada es:

$\lambda^{*}=$ Tanto anual acumulativo de crecimiento nominal de las pensiones, que se supone coincidente con el IRP.

$W=$ Edad límite de la tabla de mortalidad utilizada.

$W_{R j}=$ Pensión de jubilación inicial.

\subsection{Hipótesis de trabajo}

Con el objetivo de analizar la viabilidad financiera del sistema de pensiones español, se han introducido las probabilidades de transición de los estados laborales obtenidas y que se muestran en los Cuadros 3 y 4 . Se entiende que éstas se mantienen constantes a lo largo de todo el periodo de proyección, siendo conscientes de que se trata de un escenario extremo y pesimista y que podrá mejorar a lo largo del periodo estudiado, pero el objetivo principal de este trabajo es la inclusión de probabilidades de transición del mercado laboral en el análisis del sistema de pensiones y comprobar qué podría ocurrir en el caso de persistir esta situación de crisis económica.

Por lo que se trata de estudiar el sistema de pensiones español después de sus reformas en 2011 y en 2013 para diferentes transiciones en cuanto a mantenerse en el empleo a tiempo completo (EC) y en el tiempo parcial (EP), transitar hacia el desempleo contributivo (D) o hacia la inactividad (I), a partir de las cuáles se han definido diferentes tipos trayectorias laborales para un individuo que tiene 25 años de edad en el año 2016, tal y como se muestra en el cuadro siguiente: 


\section{Cuadro 5. Tipos de trayectorias laborales en función de las probabilidades de transición entre los estados laborales 10}

\begin{tabular}{|ccc|}
\hline Trayectorias & Situación Laboral en $\mathbf{t}$ & Situación Laboral en $\mathbf{t}+\mathbf{n}$ \\
\hline Tipo 1 & EC & EC \\
Tipo 2 & EP & EP \\
Tipo 3 & EC & EP \\
Tipo 4 & EP & EC \\
Tipo 5 & EC & D \\
Tipo 6 & EP & D \\
Tipo 7 & D & EC \\
Tipo 8 & D & EP \\
Tipo 9 & I & EC \\
Tipo 10 & I11 & EP \\
\hline
\end{tabular}

FUENTE: Elaboración propia.

En las transiciones analizadas, no se tiene en cuenta la transición desde el Empleo y el Desempleo Contributivo hacia la Inactividad en los últimos años de vida laboral, ya que para tener derecho a una pensión contributiva de jubilación, según la Ley 27/2011, el periodo mínimo de contribución es de 15 años, de los cuáles dos años deben ser inmediatamente anteriores al momento de la jubilación, por lo que aquellos individuos que transiten hacia la Inactividad, en muchos casos, pierden el derecho a la pensión contributiva.

Igualmente, no se considera la posibilidad de mantenerse en el Desempleo Contributivo al tratarse de un estado temporal, ya que según el Real Decreto Legislativo 1/1994 el periodo máximo para cobrar una prestación contributiva por Desempleo es de 2 años, siempre y cuando se tenga un periodo de cotización anterior de al menos 6 años, por ello y una vez que se sobrepasan los 2 años se considera que se transita hacia la Inactividad al no aportar contribuciones al sistema de la Seguridad Social.

10.- Para cada caso se utilizan las probabilidades de transición entre los estados laborales recogidas en los Cuadros 3 y 4 , en función de la situación de partida y de la edad de los individuos analizados en la ECV.

11.- Desde la situación de "Inactividad" sólo sería posible la transición hacia el "Empleo a Tiempo Completo" y a "Tiempo Parcial", ya que no sería posible pasar a una situación de "Desempleo contributivo". 


\subsubsection{Hipótesis relativas al cálculo de las bases de cotización}

- A partir de las rentas medias anuales ${ }^{12}$ por trabajador del año 2014, obtenidas de La Encuesta de Condiciones de Vida del INE (2011-2014), se obtienen las bases de cotización por trabajador y por edad.

- Se han calculado sus bases de cotización futuras, a partir del IRSAL proyectado por la Comisión Europea (2015), en los casos en los que se mantengan en el empleo a tiempo completo y a tiempo parcial, y en función del IPC proyectado (Comisión Europea, 2015) en aquellos casos en los que se transite hacia el desempleo contributivo.

Estas rentas medias anuales por trabajador, se muestran agrupadas por intervalos de edad de los individuos que cotiza al sistema de la Seguridad Social durante el año 2014 con contratos a tiempo completo, a tiempo parcial o como desempleados con prestaciones contributivas de desempleo. A medida que los individuos transitan hacia el empleo a tiempo parcial y hacia el desempleo contributivo sus bases de cotización desciende respecto a los periodos de empleo a tiempo completo.

- Las bases de cotización anuales ${ }^{13}$ estimadas están topadas por las cuantías máximas y mínimas vigentes en cada año en función el empleo sea a tiempo completo o parcial, o por lo contario se trate de prestaciones por desempleo contributivo.

\subsubsection{Hipótesis relativas al cálculo de las pensiones}

- En el cálculo de la cuantía de la pensión inicial se sigue las reglas establecidas en la Ley 27/2011 sin tener en cuenta el período transitorio. Se considera la jubilación a la edad legal de los 67 años. No obstante, para aquellos individuos que entre los 65 y 67 años de edad, alcancen los 38,5 años de cotización efectiva se ha supuesto que acceden a la jubilación percibiendo el $100 \%$ de su base reguladora.

- Si en algunos de los 25 años que se toman en el cálculo de la base reguladora aparecen periodos durante los cuales no se hubiesen producido cotizaciones, dichas lagunas se integran siguiendo las reglas de la Ley 27/2011.

12.- Según la ECV se entiende por renta del trabajador la remuneración monetaria que debe pagar un empleador a su asalariado como contrapartida del trabajo realizado por éste durante el periodo de referencia. Se desglosa en renta bruta monetaria o cuasimonetaria y en las cotizaciones sociales a cargo del empleador.

- La renta bruta monetaria o cuasimonetaria, se refiere a la remuneración de los asalariados. Incluye el valor de todas las cotizaciones e impuestos sobre la renta que el asalariado debe pagar a los sistemas de seguros sociales o autoridades fiscales.

-Por cotizaciones sociales a cargo del empleador, se entiende a los pagos realizados por éste durante el periodo de referencia en beneficio de sus asalariados, y se incluyen las cotizaciones a sistemas de protección social (Seguridad Social).

13.- En el cálculo de las bases de cotización no se ha tenido en cuenta la elevación de los topes de cotización establecido por el Real Decreto-ley 3/2016. 
- La primera pensión de los trabajadores a tiempo parcial, se obtiene a partir de las reglas establecidas para su cálculo en la Ley 1/2014, de 28 de febrero, para la protección de trabajadores a tiempo parcial y otras medidas urgentes del orden económico y social.

- Una vez calculada la pensión inicial, ésta se multiplica por el Factor de Sostenibilidad, obtenido a partir de la Ley 23/2013 y de las tablas de mortalidad más actuales de la Seguridad Social.

- El incremento de la pensión inicial se realiza en función del Índice de Revalorización de las Pensiones (IRP) estimado a medio plazo, coincidente con la revalorización mínima según la Ley 23/2013 (0,25\%), según las previsiones del Ministerio de Empleo y Seguridad Social (2017).

- Las pensiones estimadas se topan por las cuantías máximas y mínimas vigentes en cada momento.

\subsubsection{Hipótesis relativas al cálculo del valor actuarial de las cotizaciones y pensiones.}

- Las cotizaciones y las pensiones se valoran en términos reales al año 2017 en función del IPC, cercano al 2\%, previsto por la Comisión Europea (2015).

- El flujo de cotizaciones anuales se obtiene multiplicando el tipo de cotización aplicable a la contingencia de jubilación por las bases de cotización en términos reales obtenidas para el año 2017. Puesto que en el sistema español de pensiones contributivas la cotización total por contingencias comunes no tiene una asignación legalmente establecida para jubilación, para estimar el porcentaje de cotizaciones que se destina a la jubilación hay dos metodologías:

- Considerar que el porcentaje de cotizaciones que se destina a la jubilación es la proporción que supone el total de gastos en pensiones de jubilación, sin incluir las que proceden de incapacidad, sobre el total de gastos en pensiones del sistema.

- Considerar que el porcentaje de cotizaciones que se destina a la contingencia de jubilación es la proporción que supone el total de gastos en pensiones de jubilación, sin incluir las que proceden de incapacidad, sobre el total de ingresos por cotizaciones del sistema.

A pesar de que históricamente se ha obtenido el tipo de cotización total del sistema como una media ponderada según la cuantía de las cotizaciones. En nuestro caso, y a partir de los datos del año 2017, se ha tenido en cuenta el déficit actual del sistema, y se ha estimado un tipo de cotización cercano al 17\%, que es el que se utiliza y aplica en nuestro modelo. Este tipo de cotización se supone constante para todos los periodos. 


\section{5.- Resultados del análisis empírico de la viabilidad financiera del sistema de pensiones español ante el desempleo y la parcialidad}

Los resultados obtenidos en el análisis empírico y que se recogen en el cuadro siguiente, muestran que el TIR probable para un individuo de 25 años de edad en el 2017, varía en función de los diferentes tipos de trayectorias laborales, y por tanto de las probabilidades de transición entre los estados obtenidas de la ECV (2011-2014), es decir, la parcialidad en el empleo y el incremento del desempleo en las vidas laborales, tienen un efecto directo sobre la rentabilidad ofrecida por el sistema de pensiones, incrementando el TIR probable individual, y por tanto la no viabilidad del sistema detectada para el individuo analizado.

\section{Cuadro 6. TIR probable en función de los tipos de trayectorias laborales y probabilidades de transición entre los estados laborales 14}

\begin{tabular}{|lccc|}
\hline Trayectorias & Situación Laboral en $\mathbf{t}$ & Situación Laboral en $\mathbf{t}+\mathbf{n}$ & TIR \\
\hline Tipo 1 & EC & EC & $2,62 \%$ \\
Tipo 2 & EP & EP & $3,35 \%$ \\
Tipo 3 & EC & EP & $2,95 \%$ \\
Tipo 4 & EP & EC & $3,05 \%$ \\
Tipo 5 & EC & D & $3,25 \%$ \\
Tipo 6 & EP & D & $3,62 \%$ \\
Tipo 7 & D & EC & $3,11 \%$ \\
Tipo 8 & D & EP & $3,51 \%$ \\
Tipo 9 & I & EC & $3,33 \%$ \\
Tipo 10 & I & EP & $3,98 \%$ \\
\hline
\end{tabular}

FUENTE: Elaboración propia.

Según los resultados extraídos, se puede observar cómo un individuo de 25 años de edad en 2016 que accede al mercado de trabajo en este mismo año, y se jubilará a la edad legal, el sistema le concede un TIR del 2,62\%, en el caso de mantenerse en el Empleo a Tiempo Completo (Tipo 1) durante toda su vida laboral. Mientras que el mismo individuo para el caso de mantenerse en el Empleo a Tiempo Parcial (Tipo 2) durante toda su vida laboral, el TIR probable se incrementa hasta el 3,35\%, superior al crecimiento económico español a largo plazo proyectado por la Comisión Europea (3\%). 
Igualmente, se puede comprobar cómo a medida que el individuo transita desde el Empleo a Tiempo Completo al Empleo a Tiempo Parcial, el TIR probable se incrementa hasta el 2,95\% (Tipo 3), mientras que en el caso de transitar desde el Empleo a Tiempo Parcial hacia el Empleo a Tiempo Completo (Tipo 4), el TIR mejora y desciende al 3,05\% respecto al obtenido en el caso de mantenerse en la parcialidad durante toda la vida laboral, lo que indica que el Empleo a Tiempo Completo mejora la viabilidad financiera para el individuo de referencia.

En el análisis de las trayectorias laborales en función del porcentaje de episodios de Empleo a Tiempo Completo, el Empleo a Tiempo Parcial, el Desempleo Contributivo y la Inactividad a lo largo de su vida laboral, obtenidas a partir de las probabilidades de transición de la ECV $(2011-2014)^{15}$, se puede observar cómo a medida que se incrementan los episodios de Parcialidad, de Desempleo Contributivo y de Inactividad a lo largo de la vida laboral del individuo analizado, la rentabilidad ofrecida por el sistema se incrementa por encima de la frontera de solvencia, tal es así, que para el caso en el que se transita hacia el Desempleo, el TIR probable se incrementa hasta 3,25\% para el Empleo a Tiempo Completo (Tipo 5) y hasta el 3,62\%, para el caso de transitar desde la parcialidad (Tipo 6). Lo que indica la no viabilidad y el desfase entre las aportaciones realizadas a través de las cotizaciones sociales a lo largo de la vida laboral y las pensiones de jubilación que percibirán en el futuro, tal y como indican Samuelson (1958) y Aaron (1966).

Sin embargo, en el caso de las trayectorias laborales en las que se parte del Desempleo y se transita hacia el Empleo, la rentabilidad ofrecida por el sistema mejora, disminuyendo el TIR, 3,11\% para el Tipo 7 y hasta el 3,51\% para el Tipo 8, aunque la no viabilidad se acrecienta si lo comparamos con el TIR base obtenido en los casos de mantenerse en el Empleo a Tiempo Completo y a Tiempo Parcial, respectivamente.

En el caso en el que se presenten episodios de Inactividad a lo largo de la vida laboral, se obtienen las siguientes conclusiones: el TIR probable se incrementa hasta el 3,33\%, por encima de la considerada frontera de sostenibilidad, para el caso de transitar desde la Inactividad al Empleo a Tiempo Completo (Tipo 9), y hasta el 3,98\% para el caso de transitar hacia la Inactividad (Tipo 10), ya que como se puede comprobar las probabilidades de transitar desde la Inactividad hacia el Empleo son bajas, según la ECV (2011-2014).

Se debe mencionar que los resultados extraídos en cuanto al TIR probable son válidos para diferentes "tipos" de trayectorias laborales posibles para un individuo de 25 años de edad en 2016, para el que se le ha aplicado las probabilidades de transición entre los estados laborales, que suponemos se mantendrán en estos niveles en los próximos años. Los resultados podrán variar y ser inferiores respecto a la observación de individuos de otras edades, debido principalmente a que en nuestro

15.- Para un análisis más detallado consultar el epígrafe 4.1. de este trabajo. Además, se debe considerar el hecho de que las probabilidades de transición obtenidas se mantienen durante todo el periodo de proyección determinado. 
trabajo les afecta la inclusión del Factor de Sostenibilidad y del Índice de Revalorización de las Pensiones por lo que las reformas serán más efectivas para los más jóvenes que para quiénes tienen un mayor recorrido laboral.

\section{6.- Conclusiones}

El objetivo fundamental de este trabajo es el análisis de la viabilidad financiera del sistema de pensiones español, después de las reformas adoptadas en 2011 y 2013, y la reforma en cuanto al tratamiento de los trabajadores a tiempo parcial en el cálculo de sus pensiones de jubilación. Además, se tiene en cuenta probabilidades de transición entre el Empleo a Tiempo Completo y Parcial, el Desempleo Contributivo y la Inactividad según la ECV (2011-2014) del INE.

A partir de estos resultados, se puede concluir que a medida que se incrementan los episodios de Empleo a Tiempo Parcial, de Desempleo Contributivo y de Inactividad en la vida laboral de los cotizantes, la insostenibilidad detectada en el sistema se acrecienta (debido al descenso de las cotizaciones sociales). Aunque sí es cierto que debido al incremento de la parcialidad experimentada durante los últimos años de crisis económica, y a partir de la aprobación de la Ley 1/2014, un mayor número de trabajadores parciales quedarán cubiertos por prestaciones contributivas de jubilación, ya que a partir de esta Ley se han favorecido sus condiciones de acceso a la jubilación. Por otra parte, este hecho provoca un mayor desequilibrio entre los ingresos por cotizaciones y las pensiones que percibirán los trabajadores, sobre todo los que tienen episodios de parcialidad, de desempleo contributivo e inactividad en sus trayectorias laborales. Somos conscientes de que se trata de una visión pesimista en cuanto a la evolución del empleo, ya que se considera que las probabilidades de transición obtenidas durante la crisis económica se mantienen en el tiempo, por lo que a medida que mejore la transición hacia el Empleo a Tiempo Completo los resultados obtenidos de TIR y por tanto, la viabilidad del sistema mejorará aunque previsiblemente no se alcanzará el equilibrio financiero perseguido.

Se puede afirmar que las medidas que se adopten en el futuro con el objeto de reducir la tasa de desempleo e incrementar el empleo a tiempo completo, afectarán de forma directa a la viabilidad del sistema de pensiones y, por tanto, a la decisiones individuales en cuanto a la jubilación.

Como futura línea de investigación se presentarán los resultados de las probabilidades de transición y de TIR, tanto para hombres como para mujeres, con el objetivo de comprobar y analizar la brecha de género en las carreras laborales y sobre sus pensiones futuras. Además, de poder trabajar con las historias laborales reales que nos facilita la Muestra Continua de Vidas Laborales (MCVL) del Ministerio de Empleo y Seguridad Social. 


\section{7.- Bibliografía}

AARON, H. (1966): "The social insurance Paradox", Canadian Journal of Economic Review, vol. XXX, 3: $371-374$.

ALONSO, D. \& PAZOS, M. (2010): "Equidad y eficiencia en el sistema español de pensiones: una revisión crítica", Papeles de Trabajo del Instituto de Estudios Fiscales, P.T.N. 4/10.

ALONSO, J. \& ROSADO, B. (2016): "The effect of labour transitions in public pension financing: a case study for Spain". Disponible en: https://ssrn.com/abstract=2914691

AUTORIDAD INDEPENDIENTE DE RESPONSABILIDAD FISCAL (AIREF) (2015): "El Índice de Revalorización de las Pensiones (IRP): Propuestas de solución del problema de circularidad". (marzo 2015). Disponible en: http://www.airef.es/system/assets/archives/000/000/883/original/15.03.30_-_DT1-15-Problema_de_la_circularidad_del_IRP.pdf?1437060826

BANCO DE ESPAÑA (Ed.) (2011): Informe anual del Banco de España 2010, Madrid.

BLASCO, I. (2013): "Disfunciones en el sistema español de pensiones". En: Pensiones, Una reforma medular, Fundación de Estudios Financieros, 83-96.

CABEZAS, A. (2012): "El contrato a tiempo parcial en España y en la Comunidad de Madrid: Análisis Econométrico (2005-2008)", Revista de Economía Crítica, 14, 148-1747.

CEBRIÁN, I., MORENO, G. \& TOHARIA, L. (2009): “¿Por qué no reducen las bonificaciones la temporalidad?", Jornadas de Economía Laboral, Universidad de Zaragoza.

COMISIÓN EUROPEA (Ed.) (2015): The 2015 Ageing Report: Economic and budgetary projections for the 28 EU Member States, 2013-2060, Bruselas.

CONDE-RUIZ, J.I. \& GONZÁLEZ, C.I. (2013): "Reforma de pensiones 2011 en España", Hacienda Pública Española, 204 (1/2013), 9-44.

CONDE-RUIZ, J.I. (2013): “¿Injusticia? Los ricos soportan cada vez más la subida de las pensiones de todos". Disponible en: http://www.invertia.com/noticias/injusticia-ricos-soportan-cada-subidapensiones-2904135.htm (24 de septiembre de 2013).

CLINGMAN, M., BURKHALTER, K. \& CHAPLAIN, C. (2014): Money's worth ratios under the OASDI program for hypothetical workers, Office of the Actuary, Social Security Administration, Actuarial Note, 2014.7.

DE LA FUENTE, A. \& DOMÉNECH R. (2013): "The financial impact of Spanish pension reform: A quick estímate", Journal of Pension Economics and Finance, 12 (1), 111-137. 
DEVESA, J.E., LEJÁRRAGA, A. \& VIDAL, C. (2002): "El tanto de rendimiento de los sistemas de reparto", Revista de Economía Aplicada, vol. X, 30, 109-132.

DEVESA, J.E. \& DEVESA, M. (2010): "The cost and actuarial imbalance of pay as you go Systems: the case of Spain", Journal of Economic Policy Reform, 13(3), 259-276.

DEVESA, J.E., DEVESA, M., ENCINAS, B., DOMÍNGUEZ, I., NAGORE, A. \& MENEU, R. (2011): "Cuánto mejorará la sostenibilidad del sistema de pensiones de jubilación de la Seguridad Social tras la reforma de 2011", Asociación Española de Salud y Seguridad Social, 69-82.

DEVESA, J.E; DEVESA, M; ENCINAS, B; DOMINGUEZ, I. y MENEU, R.:

_ (2013a): Comparación entre el FEl y el FS propuesto por el Ministerio, Disponible en: www.uv.es/pensiones (16 de septiembre de 2013).

_ (2013b): ¿Qué hubiera pasado hasta 2012 con el poder adquisitivo, según el IRP?, Disponible en www.uv.es/pensiones (25 de septiembre de 2013).

_ (2013c): ¿Qué techo tendría que haberse aplicado en el IRP para que el poder adquisitivo no disminuyera en el período 1997-2012?, Disponible en: www.uv.es/pensiones (1 de octubre de 2013).

_ (2014): "El índice de revalorización de las pensiones (IRP) y su impacto sobre el sistema de pensiones español", Revista de Economía Aplicada, Observatorio, 1-23.

DÍAZ-GIMÉNEZ, J. \& DÍAZ-SAAVEDRA, J. (2011): "Parametric reforms of the spanish pension system: a quantitative analysis". Mimeo http://javierdiazgimenez.com/res/pen3-a12.pdf.

DOMÍNGUEZ, I. \& ENCINAS, B. (2008): "Inmigración y solvencia financiera del sistema público de pensiones tras la regularización de 2005", Revista de Economía Aplicada, vol. XVI, nª E-1, 67-92.

DOMínGUEZ, I., DEVESA, J., DEVESA, M., ENCINAS, B., MENEU, R. \& NAGORE, A. (2011): ¿Necesitan los futuros jubilados complementar su pensión? Análisis de las reformas necesarias y sus efectos sobre la decisión de los ciudadanos, Barcelona: Fundación Edad \& Vida.

HERCE, J.A. (2013): "Pension reform in Spain: Introducing the sustainability factor", Spanish Economic and Financial Outlook (SEFO), vol. 2, 4: 5-12.

HOYO, A. (2014): "El Factor de Sostenibilidad del sistema público de pensiones y su entrada en vigor. El factor de equidad Intergeneracional ajustado a la edad de acceso a la jubilación", Economía Española y Protección Social, IV: 75-117.

INSTITUTO NACIONAL DE ESTADÍSTICA: Encuesta de Condiciones de Vida (2011-2014).

JIMÉNEZ, S. (2013): "Envejecimiento, crisis económica y reforma del sistema de pensiones", Informe IEB sobre Federalismo Fiscal y Finanzas Públicas, 13, 54-57.

JIMENO, J.F. \& LICANDRO, O. (1999): "La tasa interna de rentabilidad y el equilibrio financiero del sistema español de pensiones de jubilación", Investigaciones Económicas, vol. XXIII, 1: 129-143. 
JIMENO, J.F. (2003): "La equidad intrageneracional de los sistemas de pensiones", Revista de Economía Aplicada, 33. Vol. XI: 5-48.

KUGLER, A., JIMENO, J. \& HERNANZ, V. (2002): "Employment consequences of restrictive permanent contracts: evidence from Spanish labour market reforms?", IZA Discussion paper, 657.

LEIMER, D.R. (1994): "Cohort-specific measures of lifetime Social Securities taxes and benefits", ORES Working Paper, No.110.

LLORENTE, R., SÁEZ, F. \& VERA, J. (2009): "Dinámica de la inserción laboral: un análisis basado en la explotación de microdatos", Jornadas de Economía Laboral, Universidad de Zaragoza.

MALO, M.A. \& TOHARIA, L. (1999a): Costes de despido y creación de empleo, Ministerio de Trabajo, Madrid.

MALO, M.A. \& TOHARIA, L. (1999b): "Costes de despido y creación de empleo en España", Economistas, 80, 308-316.

MALO, M.A. \& TOHARIA, L. (2009): “¿Qué se puede esperar de las reformas del mercado de trabajo?" Circunstancia, 20, Instituto Universitario de Investigación Ortega y Gasset.

MARSTON, S.T., FEDSTEIN, M. \& HYMANS, S.H. (1976): "Employment Instability and High Unemployment Rates", Brookings Papers on Economic Activity, Vol. 1976 1:169-210.

MENEU, R., DEVESA, J.E., DEVESA, M., NAGORE, A., DOMÍNGUEZ, I. \& ENCINAS, B. (2013): "EI Factor de Sostenibilidad: diseños alternativos y valoración financiero-actuarial de sus efectos sobre los parámetros del sistema", Economía Española y Protección Social, V: 63-96.

MURPHY, K. \& WELCH, F. (1998): "Perspectives on the social security crisis and proposed solution", American Economic Review, 88(2), 142-150.

OSFI (2012): Measuring the financial sustainability of the Canada Pension Plan, Actuarial study, No.10. Ottawa, Office of the Superintendent of Financial Institutions, Canada-Office of the Chief Actuary.

ROCH, O., BOSH, M., MORILLO, I. \& VILALTA, D. (2017): "A revision of the Revaluation Index of Spanish Pensions", Hacienda Pública Española / Revista de Economía Política.

ROSADO, B. \& DOMÍNGUEZ, I. (2014): "Solvencia financiera y la equidad del sistema de pensiones español tras las reformas de 2011 y 2013", Anales del Instituto de Actuarios Españoles, Tercera Época, 20: 122-163.

ROSADO, B., DOMÍNGUEZ, I. \& ALONSO, J.J. (2015): "Análisis empírico de la solvencia financiera y de la equidad del sistema de pensiones de jubilación español desde la perspectiva del empleo", Ekonomiaz, 88, $2^{\circ}$ semestre, 344-366.

SAMUELSON, P. (1958): "An exact consumption- Loan model of interest with or without the social contrivance of Money", The Journal of Political Economy, vol. 66, 6: 467-482. 
SÁNCHEZ, A.R. (2014): "The automatic adjustment of pension expenditures in Spain: an evaluation of the 2013 pension reform", Documentos de Trabajo, 1420, Banco de España.

TOHARIA, L. (1998): El mercado de trabajo en España, McGraw-Hill, Madrid.

VIDAL, C. (2014): "An assessment of the 2011 Spanish Pension Reform using the swedish system as benchmark", Journal of Pension Economics and Finance, 13(3), 297-333. 